\title{
Tear Osmolarity and Matrix Metallopeptidase-9 in Dry Eye Associated with Sjögren's Syndrome
}

\author{
Kyung Yun Kook, Rujun Jin, Lan Li, Hyeon Jeong Yoon, Kyung Chul Yoon \\ Department of Ophthalmology, Chonnam National University Hospital, Chonnam National University Medical School, \\ Gwangju, Korea
}

Purpose: To evaluate the correlations between tear osmolarity and matrix metallopeptidase-9 (MMP-9) and dry eye (DE) indices in patients with DE associated with Sjögren's syndrome (SS).

Methods: Sixty-three patients with DE associated with SS who underwent tear analysis were included. DE tests performed were ocular surface disease index, tear break-up time, Schirmer's test, ocular staining score, and tear osmolarity and MMP-9 tests. Correlations between tear osmolarity and DE indices, differences between patients with abnormal and normal tear osmolarity, and those between positive and negative MMP-9 patients were analyzed. Patients were classified into four groups according to tear osmolarity and MMP-9 results, and between-group differences were analyzed (group 1: abnormal tear osmolarity, MMP-9 positive; group 2: abnormal tear osmolarity, MMP-9 negative; group 3: normal tear osmolarity, MMP-9 positive; group 4: normal tear osmolarity, MMP-9 negative).

Results: Mean age of patients was $54.2 \pm 13.9$ years, and $96.2 \%$ were female. Thirty-five patients had abnormal tear osmolarity and 40 patients were MMP-9 positive. DE indices differed between groups with abnormal and normal tear osmolarity $(p<0.01)$, but not between positive and negative MMP-9 groups. There were 22 patients in group 1, 13 in group 2, 18 in group 3, and 10 in group 4. Compared to group 4, tear break-up time was shorter in groups $1(p<0.01)$ and $2(p=0.02)$. Schirmer's test values in group 1 were lower than those in group $4(p=0.03)$. Ocular staining score was higher in groups $1(p<0.01)$ and $2(p<0.05)$ than in group 4.

Conclusions: Tear osmolarity was correlated with ocular surface indices in DE associated with SS. Combination of tear osmolarity and MMP-9 test results may be helpful to determine the severity of DE associated with SS.

Key Words: Dry eye syndromes, Matrix metallopeptidase-9, Sjogren's syndrome, Tear osmolarity

Sjögren's syndrome (SS), with an estimated prevalence of $0.5 \%$ to $1.0 \%$ in the general population, is a chronic autoim-

Received: December 21, 2019 Final revision: January 15, 2020

Accepted: January 18, 2020

Corresponding Author: Kyung Chul Yoon, MD, PhD. Department of Ophthalmology, Chonnam National University Hospital, 42 Jebong-ro, Dong-gu, Gwangju 61469, Korea. Tel: 82-62-220-6741, Fax: 82-62-2271642,E-mail: kcyoon@jnu.ac.kr mune disease characterized by the production of autoantibodies, deposition of immune complexes, and infiltration of the musculoskeletal system, respiratory system, kidneys, nervous system, and exocrine glands with immune cells [1-5]. SS occurs predominately in middle-aged women and can lead to severe dry eye (DE) or dry mouth as it typically affects exocrine glands - mainly the lacrimal and salivary glands.

In SS, tear production is reduced due to chronic autoim- 
mune inflammation of the lacrimal gland [6-8]. The hyperosmolarity and resulting tear film instability increase secretion of inflammatory cytokines such as interleukin (IL)1, IL-6, tumor necrosis factor- $\alpha$, and chemokines by immune cells [9-11]. Cytokines and chemokines promote synthesis of matrix metalloproteinase-9 (MMP-9) and increase levels of Th-1 and Th-17 cells, which in turn leads to aggravation of DE symptoms via inflammation of the ocular surface, loss of epithelial cells, and reduced mucin secretion $[12,13]$. Therefore, DE associated with SS is associated with more severe clinical symptoms and signs than non-SS DE $[14,15]$.

Various conventional methods, including ocular surface disease index (OSDI), tear volume tests, tear film stability tests, and fluorescein staining have been used in the diagnosis of DE, but interpretation of the results of these tests depends on the subjective judgment of the examiner [16]. Efforts have been made to find more accurate methods that are based on the immunopathogenesis of DE, and devices that can measure tear osmolarity and detect abnormal MMP-9 have recently become commercially available.

Devices than can measure tear osmolarity and MMP-9 level are likely to be more objective than previous tests. Tear hyperosmolarity was found to be significantly correlated with the pathophysiology and severity of immune-mediated DE, such as that in SS [17-19]. Levels of MMP-9, which are known to be increased in ocular injuries such as graft-versus-host-disease and laser surgery, are also elevated in the salivary and lacrimal glands of SS patients [20-22]. Therefore, in this study, we aimed to determine whether tear osmolarity and MMP-9 measurements could supplement conventional tests in SS DE, and whether combining these two tests had utility.

\section{Materials and Methods}

\section{Ethics statement}

Ethical approval was obtained from the institutional review board of Chonnam National University Hospital (CNUH-2019-368), and the study protocol followed the guidelines of the Declaration of Helsinki. Participants were recruited from the Department of Ophthalmology, Chonnam National University Hospital. Informed consent was waived due to the retrospective nature of the study.

\section{Study population}

Subjects consisted of patients with SS who had been referred for collaborative treatment from the Department of Rheumatology between August 2017 and July 2018. SS was diagnosed based on the 2016 diagnostic criteria of the American College of Rheumatology/European League Against Rheumatism [23]. Specifically, patients were diagnosed with SS if they scored at least 4 points combined for the following criteria: (1) 3 points for a focus score of at least 1 in salivary gland biopsy, (2) 3 points if blood tests positive for SS-A antibody, (3) 1 point for a positive keratoconjunctival staining test, (4) 1 point for a positive Schirmer's test, and (5) 1 point for a positive test for salivary flow rate. Among patients with SS, we excluded patients who had a history of contact lens usage, took anti-glaucoma eye drops or systemic medications that could affect DE, or had undergone ocular surgery or trauma.

\section{Measurement of clinical ocular surface indices}

For all patients, sex, age at diagnosis, systemic disease, other ophthalmological diseases, visual acuity, and intraocular pressure were investigated. In addition, OSDI, tear break-up time (TBUT), Schirmer's test, and keratoconjunctival staining were assessed.

At each patient's first visit, the extent of symptoms was assessed using the OSDI questionnaire. The OSDI questionnaire comprises five questions on DE symptoms, four questions identifying the extent of impairment in daily living, and three questions about environmental stimuli. The overall OSDI score, which ranges from 0 to 100 , was calculated by dividing the sum of the scores for each question by the total number of questions answered [24].

To measure TBUT, one drop of $1 \%$ fluorescein solution was instilled to the inferior palpebral conjunctiva and after having the patient blink several times, the time until a defect developed in tear film fluorescence was noted. Three measurements were made under cobalt blue illumination with a slit-lamp microscope, and the mean of the three measurements was calculated. For Schirmer's test, a Schirmer's test strip (Eagle Vision, Memphis, TN, USA) was placed against the inferior palpebral conjunctiva at the lateral $1 / 3$ point, without anesthesia, and the length of the paper moistened by tears after 5 minutes was measured in millimeters. For keratoconjunctival staining, $4 \mu \mathrm{L}$ of $1 \%$ 
fluorescein solution and $4 \mu \mathrm{L}$ of $1 \%$ lissamine green were mixed and instilled; the results were scored based on the criteria of the Sjögren's International Collaborative Clinical Alliance [25]. Based on the fluorescein stain, the extent of corneal epithelial injury (0-6) was scored as 0 if there were no punctate epithelial erosions, 1 if there were $1-5$ erosive lesions, 2 if there were 6-30 erosive lesions, and 3 if there were $>30$ erosive lesions. In addition, 1 point each was added if confluent fluorescein staining was observed, if the stain was observed over the pupil, or if at least 1 filament was observed. The total score was defined as the corneal staining score. Conjunctival staining based on lissamine green staining was scored as 0 if there were $0-9$ punctate erosive lesions, 1 if there were 10-32 lesions, 2 if there were 33-100 lesions, and 3 if there were $>100$ lesions. The ocular staining score (OSS) was evaluated, out of a total of 12 points, as the sum of the corneal staining score and the nasal and temporal conjunctival staining scores.

\section{Tear analysis}

Tear osmolarity was measured in each eye using Tearlab (TearLab Corp., San Diego, CA, USA). The test card was inserted into the Tearlab system pen, which was used to collect $50 \mathrm{~nL}$ of tears on the test card from the inferolateral part of the tear film. After collection, the system pen was loaded into the system to verify the measured osmolarity. Abnormal tear osmolarity was defined as a reading of $\geq 308 \mathrm{mOsm} / \mathrm{L}$ in one eye, or a difference of $\geq 8 \mathrm{mOsm} / \mathrm{L}$ between the eyes [26-28]. Since osmolarity measurement can be affected by ambient temperature or humidity, tests were performed by a single examiner in a set location to minimize error.

For MMP-9 detection, InflammaDry (Rapid Pathogen Screening Inc., Sarasota, FL, USA) was used to obtain a single measurement from one eye; the patient was not administered any eye drops for 2 hours prior to tear collection. The lower lid was everted and tears were collected from 8-10 locations. The collected sample was combined with the included test cassette, the absorbent tip was immersed in the buffer solution for 20 seconds, and the results of the test were evaluated after 10 minutes. After 10 minutes, if the MMP-9 concentration is $>40 \mathrm{ng} / \mathrm{mL}$, a red line and blue line are both observed in the result window, and the test is interpreted as positive for MMP-9 [29]. Although the intensity of the red line can vary, in this study, the appearance of a red line was interpreted as positive, irrespective of intensity.

Patients were classified into groups based on the results of the tear osmolarity and MMP-9 tests. MMP-9 positive patients with abnormal osmolarity were assigned to group 1, MMP-9 negative patients with abnormal osmolarity were assigned to group 2, MMP-9 positive patients with normal osmolarity were assigned to group 3, and MMP-9 negative patients with normal osmolarity were assigned to group 4.

\section{Statistical analysis}

PASW Statistics ver. 18.0 (SPSS Inc., Chicago, IL, USA) was used for statistical analysis. The Kolmogorov-Smirnov test was used to test the normality of the distributions of all variables; all variables showed a normal distribution. Pearson correlation analysis was used to examine the relationships between tear osmolarity and ocular indices. A $t$-tests were used to compare the normal and abnormal osmolarity groups, and the MMP-9 positive and negative groups. One-way analysis of variance (ANOVA) and Bon-

Table 1. Clinical characteristics of patients with dry eye associated with Sjögren's syndrome

\begin{tabular}{lc}
\hline Variable & Value \\
\hline Age (yr) & $54.2 \pm 13.9$ \\
Sex & \\
$\quad$ Male & $2(3.2)$ \\
Female & $61(96.8)$ \\
Medical history & \\
Hypertension & $17(27.0)$ \\
Diabetes mellitus & $8(12.7)$ \\
Clinical dry eye parameters & \\
OSDI (score) & $26.7 \pm 20.3$ \\
TBUT (sec) & $3.7 \pm 1.2$ \\
Schirmer test (mm) & $4.5 \pm 1.1$ \\
OSS & $5.6 \pm 2.6$ \\
Tear analysis & \\
Tear osmolarity (abnormal / normal) & $37(58.7) / 28(41.3)$ \\
MMP-9 (positive / negative) & $40(63.5) / 23(36.5)$ \\
\hline
\end{tabular}

Values are presented as mean \pm standard deviation or number (\%). OSDI = ocular surface disease index; TBUT = tear break-up time; OSS = ocular staining score; MMP-9 = matrix metalloproteinase-9. 

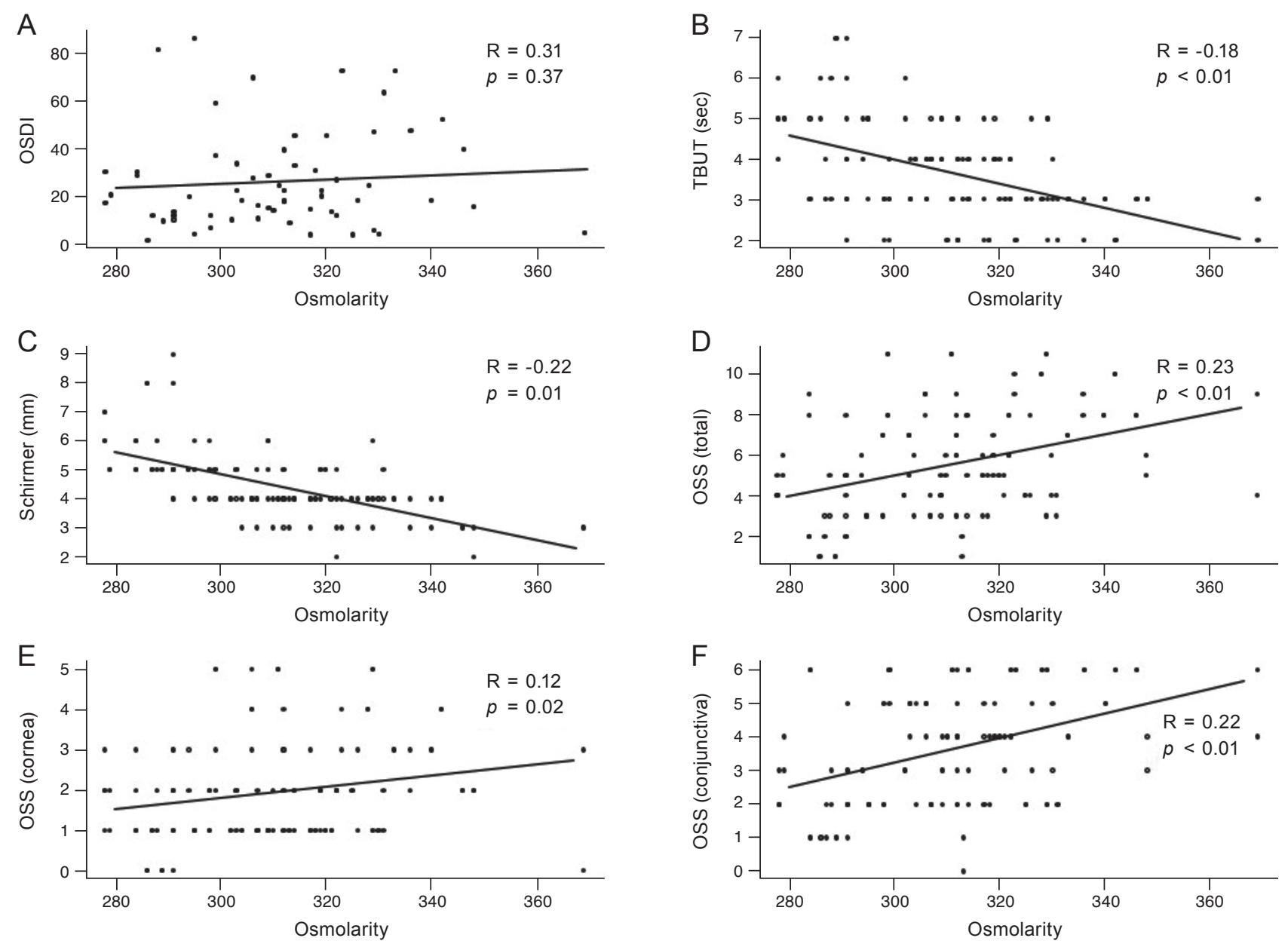

Fig. 1. Correlation between tear osmolarity level and dry eye indices. (A) No significant difference was evident between tear osmolarity and ocular surface disease index (OSDI). (B) Tear osmolarity correlated negatively with tear break-up time (TBUT), and (C) Schirmer test results, but (D) positively with ocular staining score (OSS), (E) corneal staining score, and (F) conjunctival staining score.

ferroni post-hoc corrections were used to investigate differences in DE indices between the four groups. Interaction between tear osmolarity and MMP-9 was evaluated with two-way ANOVA. In all tests, a $p$-value $<0.05$ was considered statistically significant.

\section{Results}

Eighty-six SS patients were referred from the Department of Rheumatology for collaborative treatment, of which 23 patients were excluded based on the exclusion criteria, and the remaining 63 patients were included in the study. There were two male patients (3.2\%) and 61 female patients $(96.2 \%)$, and their mean age was $54.2 \pm 13.9$ years. Thirty-seven patients (58.7\%) had abnormal tear osmolari- ty results, and 40 patients (63.5\%) were MMP-9 positive (Table 1).

Fig. 1A-1F shows the correlations between tear osmolarity and $\mathrm{DE}$ indices. Tear osmolarity was positively correlated with OSS ( $\mathrm{R}=0.23, p<0.01)$, corneal staining score ( $\mathrm{R}$ $=0.12, p=0.02)$, and conjunctival staining score $(\mathrm{R}=0.22$, $p<0.01)$. Tear osmolarity was negatively correlated with TBUT $(\mathrm{R}=-0.18, p<0.01)$ and Schirmer's test $(\mathrm{R}=-0.22$, $p=0.01)$, but showed no significant correlation with OSDI.

Comparisons between the normal and abnormal tear osmolarity groups revealed no significant difference in OSDI. The group with abnormal tear osmolarity had a shorter TBUT $(p<0.01)$, lower Schirmer's test result ( $p<$ $0.01)$, and higher OSS $(p<0.01)$ than the group with normal tear osmolarity (Table 2). There were no differences in OSDI, TBUT, Schirmer's test result, or OSS between the 
Table 2. Comparison between abnormal and normal tear osmolarity groups

\begin{tabular}{lccr}
\hline Variable & $\begin{array}{c}\text { Abnormal } \\
\text { tear osmolarity }\end{array}$ & $\begin{array}{c}\text { Normal } \\
\text { tear osmolarity }\end{array}$ & $p$-value \\
\hline Patient & $37(58.7)$ & $28(41.3)$ & \\
OSDI score & $27.4 \pm 18.8$ & $25.8 \pm 22.2$ & 0.66 \\
TBUT (sec) & $3.3 \pm 1.0$ & $4.2 \pm 1.3$ & $<0.01$ \\
Schirmer test (mm) & $4.0 \pm 0.8$ & $5.1 \pm 1.2$ & $<0.01$ \\
OSS (total) & $6.6 \pm 2.3$ & $4.6 \pm 2.6$ & $<0.01$ \\
$\quad$ Cornea & $2.2 \pm 1.1$ & $1.7 \pm 1.3$ & 0.11 \\
$\quad$ Conjunctiva & $4.4 \pm 1.4$ & $2.9 \pm 1.5$ & $<0.01$ \\
\hline
\end{tabular}

Values are presented as number (\%) or mean \pm standard deviation.

OSDI = ocular surface disease index; TBUT = tear break-up time; OSS = ocular staining score.
Table 3. Comparison between MMP-9 positive and negative groups

\begin{tabular}{lccc}
\hline Variable & MMP-9 $(+)$ & MMP-9 $(-)$ & $p$-value \\
\hline Patient & $40(63.5)$ & $23(36.5)$ & \\
OSDI score & $25.7 \pm 22.5$ & $27.5 \pm 18.6$ & 0.64 \\
TBUT (sec) & $3.9 \pm 1.5$ & $3.6 \pm 1.2$ & 0.45 \\
Schirmer test (mm) & $4.9 \pm 1.8$ & $4.5 \pm 0.8$ & 0.25 \\
$\quad<5$ / $\geq 5$ & $32 / 8$ & $13 / 10$ & 0.01 \\
OSS (total) & $6.0 \pm 2.7$ & $5.6 \pm 2.5$ & 0.92 \\
$\quad$ Cornea & $2.2 \pm 1.3$ & $1.7 \pm 1.1$ & 0.19 \\
$\quad$ Conjunctiva & $3.7 \pm 1.7$ & $3.8 \pm 1.6$ & 0.30 \\
\hline
\end{tabular}

Values are presented as number (\%), mean \pm standard deviation, or number.

MMP-9 = matrix metalloproteinase-9; OSDI = ocular surface disease index; TBUT $=$ tear break-up time; OSS $=$ ocular staining score.
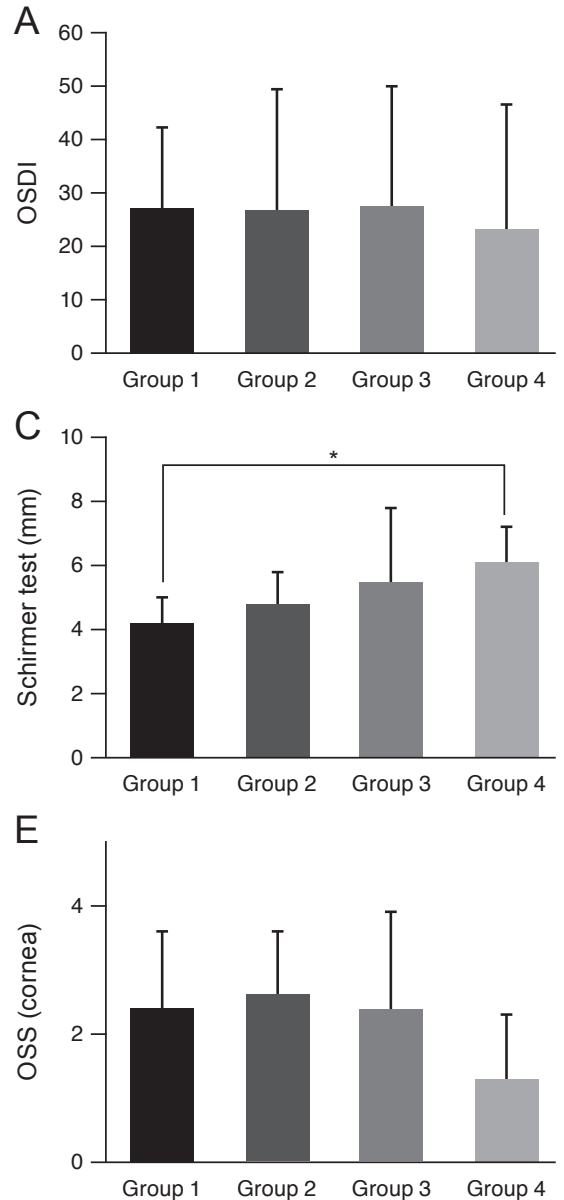
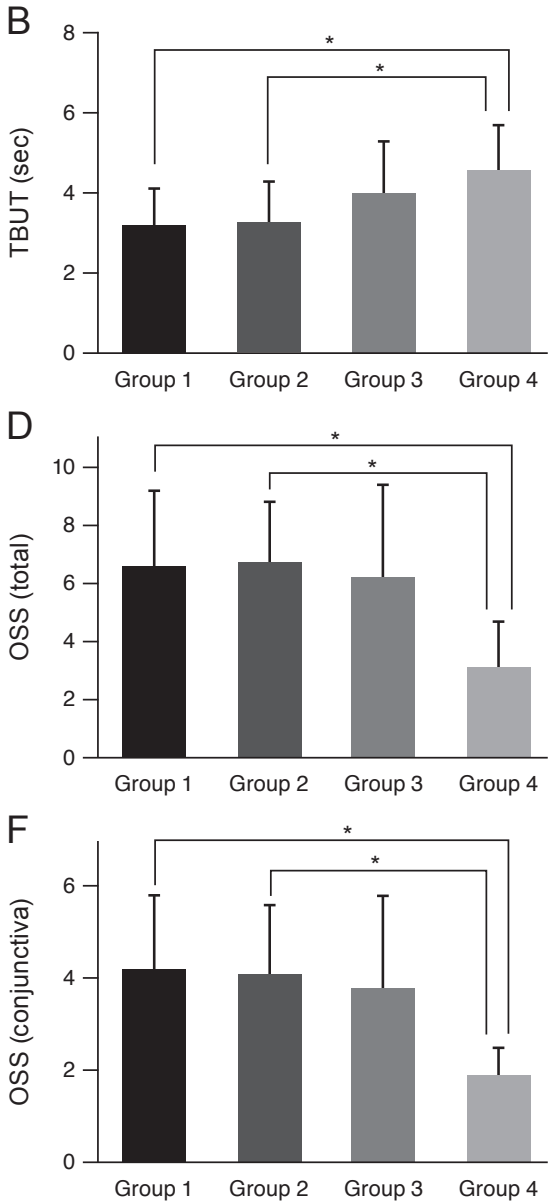

Fig. 2. Comparison of four groups classified by tear osmolarity and matrix metalloproteinase-9 (MMP-9) in (A) ocular surface disease index (OSDI), (B) tear film break-up time (TBUT), (C) Schirmer test, (D) ocular staining score (OSS), (E) corneal staining score, and (F) conjunctival staining score. Group 1: abnormal tear osmolarity and positive MMP-9, group 2: abnormal tear osmolarity and negative MMP-9, group 3: normal tear osmolarity and positive MMP-9, group 4: normal tear osmolarity and negative MMP-9. ${ }^{*} p<0.05$. 
MMP-9 positive and negative groups. However, the MMP9 positive group had a significantly higher proportion of patients with $<5 \mathrm{~mm}$ in Schirmer's test than the MMP-9 negative group (Table 3 ).

Fig. 2A-2F shows the DE indices for the four groups categorized by tear osmolarity and MMP-9 status. There were 22 patients in group $1(34.9 \%), 13$ patients in group 2 (20.6\%), 18 patients in group $3(28.6 \%)$, and 10 patients in group $4(15.9 \%)$. The four groups showed differences in TBUT ( $p<0.01)$, Schirmer's test $(p<0.01)$, and OSS ( $p<$ $0.01)$, but not in OSDI ( $p=0.57$ ). TBUT was $3.2 \pm 0.9$ seconds in group $1,3.3 \pm 1.0$ seconds in group $2,4.0 \pm 1.3$ seconds in group 3, and $4.6 \pm 1.1$ seconds in group 4; times for groups $1(p<0.01)$ and $2(p=0.02)$ were significantly shorter than the time for group 4. Schirmer's test results were $4.2 \pm 0.8 \mathrm{~mm}$ in group $1,4.8 \pm 1.0 \mathrm{~mm}$ in group 2, 5.5 $\pm 2.3 \mathrm{~mm}$ in group 3 , and $6.1 \pm 1.3 \mathrm{~mm}$ in group 4 , and the difference between group 1 and group 4 was significant ( $p$ $=0.03$ ). OSS score was $6.8 \pm 2.3$ in group $1,6.5 \pm 2.3$ in group 2, $5.0 \pm 2.9$ in group 3, and $4.1 \pm 1.8$ in group 4; values in groups $1(p=0.01)$ and $2(p<0.05)$ were significantly higher than in group 4 . Corneal staining score was not significantly different between the groups, but conjunctival staining score was significantly higher in groups $1(p=0.01)$ and $2 p<0.05)$ than in group 4 at $4.2 \pm 1.6$ in group 1, $4.1 \pm 1.5$ in group 2, $3.8 \pm 2.0$ in group 3, and $1.9 \pm$ 0.6 in group 4 . Interaction between tear osmolarity and MMP-9 was significant associated with Schirmer's test ( $p$ $<0.001$ ), but not the other DE indices.

\section{Discussion}

In this study, we performed tear osmolarity measurements and MMP-9 tests, which are closely related to the pathophysiology of SS, and analyzed their correlations with DE indices in SS patients with DE. Tear osmolarity showed statistically significant correlations with TBUT, Schirmer's test result, and OSS, but not with OSDI. The group with normal tear osmolarity and the group with abnormal tear osmolarity had significant differences in TBUT, Schirmer's test, and OSS values. MMP-9 positive and MMP-9 negative groups did not show significant differences in DE indices. When the results of the two tests were combined, the group with abnormal tear osmolarity had more advanced DE indices, regardless of MMP-9 status.
Many studies have been performed on tear osmolarity in DE since Mishima et al. [30] found increased tear osmolarity in patients with DE compared to normal subjects. Tear osmolarity shows a strong correlation with DE severity, but the diagnosis of DE is complicated due to the lack of a clear correlation between symptoms and objective clinical signs [31,32]. In our study, tear osmolarity showed significant correlations with objective DE indices such as TBUT, Schirmer's test, and OSS, but no correlations with subjective symptom score (OSDI).

In a previous study, tear osmolarity showed better ability to predict the severity of DE and diagnose DE than OSDI, TBUT, Schirmer's test, and OSS results [26]. In our study, $58.7 \%$ of subjects had abnormal tear osmolarity. This is a lower percentage of subjects than those who met the diagnostic criteria for SS, e.g., a Schirmer's test result of $<5$ $\mathrm{mm}$ (observed in $81.0 \%$ of patients) and OSS score $\geq 3$ $(88.9 \%)$. This may be because we assessed tear osmolarity only once.

Inflammation is known to activate MAPK signaling in the ocular epithelia, leading to increased MMP-9 production, and elevated MMP-9 is considered important in ocular surface diseases such as DE [33]. Messmer et al. [21] reported that MMP-9 was elevated in $40 \%$ of patients with DE disease, and correlated with not only DE tests such as OSDI, TBUT, Schirmer's test, corneal staining, and conjunctival staining, but also with meibomian gland dysfunction, female sex, and autoimmune diseases such as SS and thyroid disease. However, in another study that used an MMP-9 point-of-care test, MMP-9 levels were not correlated with the severity of ocular surface inflammation [34]. Similarly, there was no correlation between MMP-9 test results and DE indices in our study.

However, $80 \%$ of MMP-9 positive patients had a Schirmer's test result of $<5 \mathrm{~mm}$, which is a diagnostic criterion for SS, and this was a significantly higher than the proportion of MMP-9 negative patients that met this criterion (55.6\%). Thus, MMP-9 positive results were obtained in more severe $\mathrm{DE}$, and were correlated with $\mathrm{DE}$ severity. Inflammadry, which was used for MMP-9 measurement, has a sensitivity of $66 \%$ to $97 \%$ in DE patients [35]. In our study, $63.5 \%$ of patients with DE associated with SS were MMP-9 positive. In one study that used both Inflammadry and an enzyme-linked immunosorbent assay to measure MMP-9, no correlation was found between MMP-9 levels $\geq 40 \mathrm{ng} / \mathrm{mL}$ (the threshold value of InflammaDry) and DE 
indices and the sensitivity for DE diagnosis was $11 \%$, but there was a correlation between MMP-9 levels $\geq 10 \mathrm{ng} / \mathrm{mL}$ and DE symptoms and indices [36].

We classified patients into four groups based on the combined results of the tear osmolarity and MMP-9 tests, and found that, irrespective of the MMP-9 results, there were significant differences in clinical indices between groups with abnormal and normal tear osmolarity. Furthermore, there were statistically significant differences in TBUT, Schirmer's test, total OSS, and conjunctival staining score between groups 1 and 2, and group 4. Thus, combining tear osmolarity and MMP-9 results might be useful to predict the severity of DE associated with SS. Since this was a single-center, cross-sectional study in a tertiary institution, rather than an epidemiological study of the entire population, it may have been biased towards patients with more severe symptoms. Although tear osmolarity and MMP-9 were measured with specialized devices, measurements were taken only once, which limits the reliability of the test results. In addition, due to the small sample size in this study, we were unable to determine reference values for tear osmolarity in SS; large-scale studies are required to determine these.

Our study is valuable because we measured tear osmolarity and MMP-9, which are closely related to SS pathophysiology, and demonstrated their correlations with $\mathrm{DE}$ indices. Tear osmolarity was correlated with DE indices. Furthermore, combining tear osmolarity and MMP-9 test results may be helpful to determine the severity of DE associated with SS. Patients with severe DE tended to be MMP-9 positive, indicating that measurement of MMP-9 may be useful for future diagnosis and grading of DE in patients with SS.

\section{Conflict of Interest}

No potential conflict of interest relevant to this article was reported.

\section{References}

1. Thomas E, Hay EM, Hajeer A, Silman AJ. Sjogren's syndrome: a community-based study of prevalence and impact. Br J Rheumatol 1998;37:1069-76.
2. Jacobsson LT, Axell TE, Hansen BU, et al. Dry eyes or mouth--an epidemiological study in Swedish adults, with special reference to primary Sjogren's syndrome. J Autoimmun 1989;2:521-7.

3. Bjerrum KB. Keratoconjunctivitis sicca and primary Sjogren's syndrome in a Danish population aged 30-60 years. Acta Ophthalmol Scand 1997;75:281-6.

4. Jonsson R, Haga HJ, Gordon TP. Current concepts on diagnosis, autoantibodies and therapy in Sjogren's syndrome. Scand J Rheumatol 2000;29:341-8.

5. Mavragani CP, Moutsopoulos HM. Sjogren's syndrome. Annu Rev Pathol 2014;9:273-85.

6. El Annan J, Chauhan SK, Ecoiffier T, et al. Characterization of effector T cells in dry eye disease. Invest Ophthalmol Vis Sci 2009;50:3802-7.

7. De Paiva CS, Chotikavanich S, Pangelinan SB, et al. IL-17 disrupts corneal barrier following desiccating stress. $\mathrm{Mu}$ cosal Immunol 2009;2:243-53.

8. Zoukhri D. Effect of inflammation on lacrimal gland function. Exp Eye Res 2006;82:885-98.

9. Luo L, Li DQ, Doshi A, et al. Experimental dry eye stimulates production of inflammatory cytokines and MMP-9 and activates MAPK signaling pathways on the ocular surface. Invest Ophthalmol Vis Sci 2004;45:4293-301.

10. Siemasko KF, Gao J, Calder VL, et al. In vitro expanded CD4+CD25+Foxp3+ regulatory $\mathrm{T}$ cells maintain a normal phenotype and suppress immune-mediated ocular surface inflammation. Invest Ophthalmol Vis Sci 2008;49:5434-40.

11. Chauhan SK, El Annan J, Ecoiffier T, et al. Autoimmunity in dry eye is due to resistance of Th17 to Treg suppression. J Immunol 2009;182:1247-52.

12. Li DQ, Chen Z, Song XJ, et al. Stimulation of matrix metalloproteinases by hyperosmolarity via a JNK pathway in human corneal epithelial cells. Invest Ophthalmol Vis Sci 2004;45:4302-11.

13. Li DQ, Chen Z, Song XJ, et al. Hyperosmolarity stimulates production of MMP-9, IL-1ß and TNF-by human corneal epithelial cells via a c-Jun NH2-terminal kinase pathway. Invest Ophthalmol Vis Sci 2002;43:1981.

14. Goto E, Matsumoto Y, Kamoi M, et al. Tear evaporation rates in Sjogren syndrome and non-Sjogren dry eye patients. Am J Ophthalmol 2007;144:81-5.

15. Long Q, Wang JY, Xu D, Li Y. Comparison of corneal biomechanics in Sjogren's syndrome and non-Sjogren's syndrome dry eyes by Scheimpflug based device. Int J Ophthalmol 2017;10:711-6. 
16. Bron AJ. Diagnosis of dry eye. Surv Ophthalmol 2001; 45:S221-6.

17. Martinez-Carrasco R, Sanchez-Abarca LI, Nieto-Gomez C, et al. Assessment of dry eye in a GVHD murine model: approximation through tear osmolarity measurement. Exp Eye Res 2017;154:64-9.

18. Berchicci L, Iuliano L, Miserocchi E, et al. Tear osmolarity in ocular graft-versus-host disease. Cornea 2014;33:1252-6.

19. Stevenson W, Chauhan SK, Dana R. Dry eye disease: an immune-mediated ocular surface disorder. Arch Ophthalmol 2012;130:90-100.

20. Pflugfelder SC, Jones D, Ji Z, et al. Altered cytokine balance in the tear fluid and conjunctiva of patients with Sjogren's syndrome keratoconjunctivitis sicca. Curr Eye Res 1999;19:201-11.

21. Messmer EM, von Lindenfels V, Garbe A, Kampik A. Matrix metalloproteinase 9 testing in dry eye disease using a commercially available point-of-care immunoassay. Ophthalmology 2016;123:2300-8.

22. Chotikavanich S, de Paiva CS, Li de Q, et al. Production and activity of matrix metalloproteinase- 9 on the ocular surface increase in dysfunctional tear syndrome. Invest Ophthalmol Vis Sci 2009;50:3203-9.

23. Shiboski CH, Shiboski SC, Seror R, et al. 2016 American College of Rheumatology/European League Against Rheumatism classification criteria for primary Sjogren's syndrome: a consensus and data-driven methodology involving three international patient cohorts. Ann Rheum Dis 2017;76:9-16.

24. Schiffman RM, Christianson MD, Jacobsen G, et al. Reliability and validity of the ocular surface disease index. Arch Ophthalmol 2000;118:615-21.

25. Whitcher JP, Shiboski CH, Shiboski SC, et al. A simplified quantitative method for assessing keratoconjunctivitis sicca from the Sjogren's Syndrome International Registry. Am J Ophthalmol 2010;149:405-15.

26. Lemp MA, Bron AJ, Baudouin C, et al. Tear osmolarity in the diagnosis and management of dry eye disease. Am $J$ Ophthalmol 2011;151:792-8.

27. Jacobi C, Jacobi A, Kruse FE, Cursiefen C. Tear film osmolarity measurements in dry eye disease using electrical impedance technology. Cornea 2011;30:1289-92.

28. Sullivan B. Challenges in using signs and symptoms to evaluate new biomarkers of dry eye disease. Ocul Surf 2014;12:2-9.

29. Kaufman HE. The practical detection of mmp-9 diagnoses ocular surface disease and may help prevent its complications. Cornea 2013;32:211-6.

30. Mishima S, Kubota Z, Farris RL. The tear flow dynamics in normal and in keratoconjunctivitis sicca cases. In: Solanes MP, editor. Ophthalmology: proceedings of the XXI International Congress; 1970 March 8-14; Mexico. Amsterdam: Excerpta Medica; 1971. p. 1801-5.

31. Suzuki M, Massingale ML, Ye F, et al. Tear osmolarity as a biomarker for dry eye disease severity. Invest Ophthalmol Vis Sci 2010;51:4557-61.

32. Kim M, Kim HS, Na KS. Correlation between tear osmolarity and other ocular surface parameters in primary Sjogren's syndrome. Korean J Ophthalmol 2017;31:25-31.

33. Tomlinson A, Khanal S, Ramaesh K, et al. Tear film osmolarity: determination of a referent for dry eye diagnosis. Invest Ophthalmol Vis Sci 2006;47:4309-15.

34. Lanza NL, McClellan AL, Batawi H, et al. Dry eye profiles in patients with a positive elevated surface matrix metalloproteinase 9 point-of-care test versus negative patients. Ocul Surf 2016;14:216-23.

35. Sambursky R, Davitt WF 3rd, Latkany R, et al. Sensitivity and specificity of a point-of-care matrix metalloproteinase 9 immunoassay for diagnosing inflammation related to dry eye. JAMA Ophthalmol 2013;131:24-8.

36. Schargus M, Ivanova S, Kakkassery V, et al. Correlation of tear film osmolarity and 2 different MMP-9 tests with common dry eye tests in a cohort of non-dry eye patients. Cornea 2015;34:739-44. 